\title{
Prevalência de idosos restritos ao domicílio em região metropolitana de Belo Horizonte (Minas Gerais, Brasil)
}

\author{
Prevalence of housebound elderly people \\ in the urban region of Belo Horizonte (Minas Gerais, Brazil)
}

Príscila Guedes Santana Ursine ${ }^{1}$

Hésio de Albuquerque Cordeiro ${ }^{2}$

Claudia Leite Moraes ${ }^{3}$

${ }^{1}$ Faculdade Pitágoras Belo Horizonte. Rua Timbiras 1375, Funcionários. 30140060 Belo Horizonte MG. priscilaursine@yahoo.com.br ${ }^{2}$ Universidade Estácio de Sá. ${ }^{3}$ Universidade do Estado do Rio de Janeiro.

\begin{abstract}
The aim of this article is to estimate the prevalence and the socio-demographic and health profile of housebound elderly people registered at a Family Health Unit in the urban region of Belo Horizonte (Minas Gerais, Brazil). A household survey was conducted between May and July 2006 with 275 elderly people selected via cluster sampling. The svy suite of commands in Stata 9.0 was used to deal adequately with the cluster sample structure and to allow the incorporation of fractions of expansion in the analyses. Among the 275 elderly, 22.4\% (IC95\%: 14.7; 32.4) were restricted to their homes. The prevalence of this condition was greater among women, people over 80 and suspected of suffering from cognitive impairment ( $p$-valor $<0.05)$. The majority of housebound people had incomes below the minimum wage, reported history of falls, depression and indicated physical disorders as the cause of the restriction. The large contingent of low-income housebound elderly with several health problems, reinforces the need for incorporation of proposals for promotion and vigilance of the health of the elderly, which extend beyond the boundaries of the healthcare units.
\end{abstract}

Key words Housebound elderly people, Household survey, Family Health Program
Resumo Este artigo tem por objetivo estimar a prevalência e o perfil sociodemográfico e de saúde dos idosos restritos ao domicílio adscritos a uma unidade de saúde da família da região metropolitana de Belo Horizonte (Minas Gerais). Realizou-se inquérito domiciliar no período de maio a julho de 2006 com 275 idosos selecionados através de amostragem por conglomerados. Utilizou-se a suite svy do aplicativo Stata 9.0 para lidar adequadamente com a estrutura amostral de conglomeração e permitir a incorporação das frações de expansão nas análises. Dos 275 idosos entrevistados, 22,4\% (IC95\%: 14,7; 32,4) eram restritos ao domicílio. A prevalência dessa condição foi maior entre as mulheres, entre os indivíduos com 80 anos ou mais e entre aqueles com suspeita de déficit cognitivo ( $p$-valor $<0,05)$. A maior parte dos indivíduos restritos tinha renda inferior a um salário minimo, relatava história de queda, era caso suspeito de depressão e referia motivos físicos para a restrição. O grande contingente de idosos restritos ao domicílio, de baixa renda e com diferentes problemas de saúde, reforça a necessidade de incorporação de propostas de promoção e vigilância em saúde do idoso que ultrapassem as fronteiras das unidades de saúde.

Palavras chave Idosos restritos ao domicílio, Inquérito domiciliar, Programa Saúde da Família 


\section{Introdução}

Desde a década de 60 , a população brasileira assiste à sua transição demográfica, caracterizada por um acentuado processo de envelhecimento populacional. Infelizmente, o aumento da expectativa de vida nem sempre é acompanhado da melhoria da qualidade de vida, especialmente quando se considera a população de baixa renda e maior risco social ${ }^{1}$. Neste caso, a sobreposição de diferentes patologias e a insuficiência de uma abordagem integral pelos serviços de saúde fazem parte do processo de desenvolvimento de incapacidades e dependências no idoso, resultando, muitas vezes, na restrição das atividades de vida diária ao ambiente domiciliar.

Segundo alguns estudos, na maior parte das vezes esta restrição ao domicílio vem acompanhada de um alto grau de dependência dos idosos para a realização das suas atividades do dia a dia $^{2,3}$. Esta diminuição de autonomia vem chamando a atenção tanto dos profissionais de saúde como dos pesquisadores que se dedicam a este grupo populacional, já que as relações entre dependência funcional e morbimortalidade do idoso vêm sendo ressaltadas por diferentes pesquisadores ${ }^{4,5}$.

Um dos componentes do processo que une a dependência funcional ao aumento do risco de patologias e mortalidade precoce parece ser a restrição do idoso ao ambiente doméstico. Segundo alguns autores, esta dependência levaria à restrição do idoso à sua área de moradia e o subsequente desenvolvimento e/ou agravamento de patologias, reduzindo suas condições de saúde ${ }^{3,6}$. Outros autores sugerem que a restrição ao domicílio é que seria um fator de risco à incapacidade funcional progressiva ${ }^{2,7}$. Nesta perspectiva, Fujita et $a l^{2}$ indicam que a redução da frequência de sair de casa pode ocorrer concomitantemente à deterioração do estado da saúde, representando um estágio de pré-restrição ao domicílio, importante fator preditor de incapacidade funcional e morbimortalidade entre os idosos.

A restrição ao domicílio parece ser um processo insidioso e dinâmico, podendo ser modificado ou prevenido se houver ambiente e intervenção adequada em seus fatores de risco. Até o momento, pouco se sabe sobre os fatores que propiciam essa restrição em razão da escassez da literatura e sua relativa limitação aos países desenvolvidos.

Uma vez comprometida a capacidade funcional do idoso, algum tipo de suporte e auxílio é necessária, seja em âmbito institucional ou do- miciliar. A internação de idosos em asilos, casa de repouso e similares está sendo questionada mesmo nos países desenvolvidos devido aos altos custos desse modelo e sua influência negativa na independência e autonomia dos pacientes. Em contrapartida, a atenção domiciliária, oferecida por instituições públicas ou privadas, tem sido cada vez mais valorizada.

No Brasil, historicamente, o desenvolvimento da assistência domiciliária tem apresentado um caráter excludente, uma vez que, na maior parte das vezes, apenas os segurados à previdência social ou que possuam planos de saúde têm acesso a esse tipo de assistência. No entanto, o processo de construção do Sistema Único de Saúde (SUS) e, posteriormente, o desenvolvimento da Estratégia Saúde da Família (ESF) vêm transformando essa realidade. Como alternativa ao modelo assistencial tradicional, a ESF se baseia na reorganização da prática de atenção básica à saúde, tendo a visita domiciliar como instrumento de intervenção fundamental ${ }^{8}$. Desta forma, é possível que a Estratégia tenha grande potencial na atenção à saúde do idoso, particularmente aqueles incapacitados crônicos que tendem a permanecer restritos em seus lares.

Porém, pouco se tem avançado na sistematização de ações voltadas para esse subgrupo populacional. É possível que a timidez de propostas organizadas de atenção aos idosos restritos ao domicílio seja consequência da inexistência de estudos que estimem a magnitude do problema e suas principais características em nosso meio. Considerando que as particularidades do contexto brasileiro, no que tange ao processo de envelhecimento populacional ${ }^{9}$, propiciam quadros diferentes dos encontrados em países desenvolvidos, o desenvolvimento de pesquisas que possam subsidiar a elaboração das ações se torna ainda mais relevante.

Visando contribuir para a redução desta importante lacuna, o objetivo do presente estudo é estimar a prevalência e o perfil sociodemográfico e de saúde de idosos restritos ao domicílio adscritos ao PSF do Centro de Saúde Ventosa em Belo Horizonte (Minas Gerais).

\section{Métodos}

\section{Desenho e contexto do estudo}

Trata-se de um estudo transversal de base populacional, realizado no período de maio a julho de 2006 na área de abrangência do Centro de 
Saúde Ventosa (CSV), em Belo Horizonte (Minas Gerais). O CSV possui cinco equipes de saúde da família responsáveis por 24.650 indivíduos cadastrados. Destes, 1.651 (6,7\%) são idosos ${ }^{10}$.

A exemplo do que ocorre em todo o município de Belo Horizonte, a área de abrangência do Centro de Saúde Ventosa também apresenta população de diferentes estratos socioeconômicos.

\section{Estratégia de seleção dos indivíduos e tamanho amostral}

A estratégia de seleção da população do estudo seguiu as recomendações de Veras et al. ${ }^{11}$ para inquéritos domiciliares com populações idosas. Para garantir a representatividade da amostra com relação aos diferentes subgrupos populacionais, a área de abrangência do CSV foi dividida em estratos de acordo com o Índice de Vulnerabilidade Social (IVS), utilizado pela Secretaria Municipal de Saúde de Belo Horizonte ${ }^{10}$.

O IVS é um escore obtido a partir da combinação de indicadores socioeconômicos (saneamento básico, condições de habitação, escolaridade, renda, características demográficas e de saúde da família) que busca resumir informações relevantes para a identificação de desigualdades intraurbanas, apontando áreas prioritárias para intervenção e alocação de recursos. Após o cálculo do índice de vulnerabilidade à saúde, os setores foram classificados nos seguintes estratos socioeconômicos: baixo risco (setores com valores inferiores ao valor médio da população adstrita menos 1/2 desvio padrão); médio risco (setores que apresentam valor de IVS dentro do intervalo limitado pelo valor médio mais ou menos $1 / 2$ desvio padrão); elevado risco (setores com IVS entre $1 / 2$ e 1 desvio padrão acima do valor médio) e muito elevado risco (setores com valores acima dos que caracterizam uma situação de elevado risco $)^{10}$.

Dentro de cada estrato, procedeu-se o sorteio de duas microáreas (Unidades Primárias de Amostragem). Em seguida, procedeu-se à seleção de cerca de 70\% dos idosos residentes nas microáreas que pertenciam aos estratos de baixo, médio e elevado riscos. Para garantir um número suficiente de indivíduos da área de muito elevado risco, uma fração maior dos idosos desta região (90\%) foi selecionada.

Foram elegíveis para o estudo os idosos com idade igual ou superior a 60 anos que concordassem em participar da pesquisa e possuíssem condições cognitivas para responder ao questionário e/ou a presença de um familiar ou cuidador que pudesse auxiliar na resposta no ato da entrevista. O tamanho amostral final foi de 275 idosos.

\section{Coleta de dados e instrumentos de aferição}

As informações foram obtidas por meio de entrevistas face a face, realizadas pela pesquisadora principal e por quatro alunas de graduação em Fisioterapia do Centro Universitário de Belo Horizonte. Quando o idoso estava impossibilitado de responder à entrevista devido a algum problema de saúde ou déficit cognitivo, recorreu-se a um cuidador ou familiar. Nessas situações, as questões que requeriam julgamento do entrevistado foram deixadas em branco.

A coleta de dados foi realizada em duas etapas. A primeira (etapa A) visou caracterizar a população idosa cadastrada nas equipes de saúde da família com relação a sexo, faixa etária, anos de escolaridade, suspeita de déficit cognitivo e identificar os idosos restritos ao domicílio. Para isso, todos os idosos da amostra foram investigados em relação à sua mobilidade, ou seja, à capacidade de sair de casa sem acompanhante. Foi considerado "restrito ao domicílio" o indivíduo que respondeu negativamente à seguinte pergunta: $O$ Sr(a). é capaz de sair de casa sozinho(a)? Considerou-se "restrito ao leito" o indivíduo que não tinha condições de sair do leito sem ajuda de outros. Foi investigada também a causa da restrição ao domicílio segundo a percepção do idoso e/ou cuidador. Essas perguntas foram precedidas pelos dados de identificação e aplicação do Miniexame do Estado Mental (MEEM), instrumento de rastreio para avaliação cognitiva, que permite inferir sobre a presença de "provável demência"12.

A etapa B objetivou caracterizar o perfil dos idosos restritos ao domicílio através de uma avaliação multidimensional, que contemplou uma caracterização de suas condições demográficas, socioeconômicas, de saúde e funcionais. Os idosos foram agrupados em três faixas etárias (60 a 69; 70 a 79; e 80 anos ou mais). Avaliou-se a distribuição por sexo, situação conjugal, cor autorreferida e tipo de arranjo domiciliar (número de gerações residentes no domicílio). A renda familiar em salários mínimos na época e o nível de escolaridade (anos completos de escolaridade) foram utilizados para categorização da população em termos socioeconômicos. A partir da renda familiar, foi calculada a renda per capita (total da renda familiar no mês que precedeu a visita sobre o número de pessoas residentes no domicílio).

Os indivíduos restritos ao domicílio tiveram seus motivos de dependência classificados como 
físicos, cognitivos e comportamentais, de acordo com critério de Baltes ${ }^{13}$. A análise da autopercepção da saúde foi baseada na questão Em geral, como o(a) senhor(a) avalia sua saúde atualmente? As respostas variaram em uma escala de 1 a 5 ( $1=$ muito ruim; $2=$ ruim; $3=$ moderada; $4=$ boa; $5=$ muito boa $)^{14}$.

A história de quedas foi definida como relato de um ou mais episódios de queda nos últimos 12 meses. As demais patologias associadas foram registradas de acordo com o relato dos idosos e /ou cuidadores.

Para avaliação da capacidade funcional do idoso foi utilizada a dimensão relacionada ao desempenho nas Atividades Básicas de Vida Diária (ABVD) e Atividades Instrumentais de Vida Diária (AIVD) proveniente do Older Americans Resources and Service (OARS) Multidimensional Functional Assessment Questionnaire (OMFAQ) ${ }^{15}$, questionário multidimensional concebido nos Estados Unidos da América, traduzido e adaptado para o português ${ }^{1}$. A classificação da capacidade funcional foi feita de acordo com o grau de dependência nas AVDs, proposto por Ramos et $a l .^{16}$. Para avaliação do estado tímico dos idosos, utilizou-se a versão nacional da Escala Geriátrica de Depressão (GDS), instrumento desenvolvido por Sheikh e Yesavage ${ }^{17}$ que permite a detecção dos casos de "provável depressão".

\section{Análise de dados}

O processamento de variáveis e análise de dados foi realizada em Stata $9^{18}$. Na análise, utilizou-se a suíte svy do aplicativo para lidar adequadamente com a estrutura amostral de conglomeração e permitir a incorporação das frações de expansão nas análises.

A coleta de dados foi iniciada após aprovação do Comitê de Ética em Pesquisa da Secretaria Municipal de Saúde de Belo Horizonte, uma vez que respeitou os princípios éticos contidos na Declaração de Helsinque e a Resolução no 196 do Conselho Nacional de Ética em Pesquisa.

\section{Resultados}

Dos 320 idosos selecionados inicialmente para o estudo, 275 foram entrevistados. Os 14\% de perdas foram por óbito $(0,9 \%)$, mudança de endereço $(1,5 \%)$, idoso não encontrado $(7,1 \%)$ e recusa em receber pessoas estranhas $(4,5 \%)$.

Como apresentado na Tabela 1, a maioria dos respondentes era do sexo feminino (63,6\%;
IC95\%: 56,4-70,2), tinha menos quatro anos de estudo (52,1\%; IC95\%: 43,5-60,6) e apresentava estado cognitivo normal (89,9\%; IC95\%: 72,896,7). Com relação à faixa etária, nota-se que grande parte dos indivíduos entrevistados tinha entre 60 e 69 anos (45,5\%; IC95\%: 39, 0-52,1). No momento do estudo, estimou-se que $22,4 \%$ (IC95\%: 14,7-32,4) dos idosos da comunidade se encontravam restritos ao domicílio.

A distribuição das características demográficas, socioeconômicas e de saúde dos 68 idosos restritos ao domicílio está apresentada na Tabela 2. Em termos sociodemográficos, observa-se grande proporção de mulheres com oitenta anos ou mais, viúvas, que se autorreferem como de cor parda, vivendo com duas ou mais gerações, com baixa escolaridade e renda per capita de até um salário mínimo.

Em relação à caracterização da saúde desses idosos, nota-se que quase $50 \%$ da amostra percebem sua saúde como "regular". A maioria apresentava estado cognitivo normal, referia história de queda alguma vez na vida, apresentava sinais

Tabela 1. Caracterização da população de idosos do Centro de Saúde Ventosa - Belo Horizonte (MG, Brasil), 2006.

\begin{tabular}{|c|c|c|}
\hline Características dos idosos & $(\%)^{*}$ & IC $95 \%{ }^{* *}$ \\
\hline \multicolumn{3}{|l|}{ Sexo } \\
\hline Feminino & 63,6 & $56,4-70,2$ \\
\hline Masculino & 36,5 & $29,8-43,6$ \\
\hline \multicolumn{3}{|l|}{ Faixa etária (em anos) } \\
\hline $60-69$ & 45,5 & $39,0-52,1$ \\
\hline $70-79$ & 37,3 & $28,6-46,9$ \\
\hline $80 \mathrm{ou}+$ & 17,3 & $14,6-20,2$ \\
\hline \multicolumn{3}{|l|}{ Anos completos de escolaridade } \\
\hline Nunca frequentou escola & 26,9 & $20,8-33,9$ \\
\hline De um a três anos & 25,2 & $17,5-34,8$ \\
\hline De quatro a sete anos & 26,6 & $19,0-35,9$ \\
\hline Oito ou mais & 21,3 & $16,7-26,7$ \\
\hline \multicolumn{3}{|l|}{ Suspeita de déficit cognitivo ${ }^{* *}$} \\
\hline Ausente & 89,9 & $72,8-96,7$ \\
\hline Presente & 10,1 & $3,3-27,1$ \\
\hline \multicolumn{3}{|l|}{ Nível de restrição da locomoção } \\
\hline Não é restrito & 68,0 & $54,8-78,9$ \\
\hline Restrito à vizinhança & 9,6 & $4,3-20,3$ \\
\hline Restrito à residência & 18,6 & $12,8-26,3$ \\
\hline Restrito ao leito & 3,8 & $1,9-7,5$ \\
\hline \multicolumn{3}{|c|}{$\begin{array}{l}\text { "\% estimado após expansão da amostra; }{ }^{* *} \text { Intervalo de confiança a } \\
95 \% \text {, considerando-se a estratificação de acordo com o IVS, as } \\
\text { microáreas (MA) como Unidades Primárias de Amostragem } \\
\text { (UPA) e o fator de expansão de cada indivíduo; } \\
\text { do Miniexame do Estado Mental (MEEM), considerando-se como } \\
\text { ponto de corte } 13 \text { pontos. }\end{array}$} \\
\hline
\end{tabular}


indicativos de depressão e identificava o motivo da restrição como de natureza física. Dentre as patologias formalmente investigadas durante a entrevista, a hipertensão arterial sistêmica revelou-se o agravo mais prevalente. No entanto, de acordo com os idosos, o acidente vascular ence- fálico (AVE), os problemas de coluna, de memória e a incontinência urinária são os que mais interferem em suas atividades diárias. Em termos dos agravos físicos que motivavam a restrição ao domicílio, o AVE e as alterações na acuidade visual foram os mais comentados.

Tabela 2. Distribuição das características demográficas, socioeconômicas e de saúde dos idosos restritos ao domicílio e/ou ao leito - Belo Horizonte (MG, Brasil), 2006.

\begin{tabular}{|c|c|c|c|}
\hline Características & $\mathbf{n}^{*}$ & $\%^{* *}$ & IC95\% ${ }^{* * *}$ \\
\hline Sexo & & & \multirow{3}{*}{$\begin{array}{l}(61,8-81,6) \\
(18,4-38,2)\end{array}$} \\
\hline Feminino & 50 & 72,8 & \\
\hline Masculino & 18 & 27,2 & \\
\hline Faixa etária & & & \multirow{4}{*}{$\begin{array}{l}(12,5-54,6) \\
(13,9-45,2) \\
(36,4-51,8)\end{array}$} \\
\hline 60-69 & 20 & 29,3 & \\
\hline $70-79$ & 25 & 26,7 & \\
\hline $80 \mathrm{ou}+$ & 23 & 43,9 & \\
\hline Situação conjugal & & & \multirow{4}{*}{$\begin{array}{r}(24,4-65,6) \\
(24,3-47,2) \\
(9,7-39,9)\end{array}$} \\
\hline Viúvo & 29 & 44,0 & \\
\hline Casado/amasiado & 27 & 34,9 & \\
\hline Solteiro/separado/divorciado & 12 & 21,1 & \\
\hline Cor & & & \multirow{4}{*}{$\begin{array}{l}(32,2-54,9) \\
(28,1-55,2) \\
(10,3-23,6)\end{array}$} \\
\hline Parda/mulata/morena/cabocla & 31 & $43,2 \%$ & \\
\hline Branca & 25 & $40,9 \%$ & \\
\hline Negra & 11 & $15,9 \%$ & \\
\hline Tipo de arranjo domiciliar & & & \multirow{3}{*}{$\begin{array}{r}(2,7-49,6) \\
(50,4-97,3)\end{array}$} \\
\hline Vive só ou com pessoas da mesma geração & 11 & 14,2 & \\
\hline Vive com duas gerações ou mais & 57 & 85,8 & \\
\hline Anos completos de escolaridade & & & \multirow{4}{*}{$\begin{array}{r}(19,6-67,5) \\
(28,8-63,4) \\
(6,0-25,5)\end{array}$} \\
\hline Nunca frequentou escola & 31 & 41,6 & \\
\hline De um a sete anos & 29 & 45,6 & \\
\hline Oito ou mais & 6 & 12,8 & \\
\hline Renda per capita (em salários mínimos) & & & \multirow{4}{*}{$\begin{array}{r}(57,2-79,0) \\
(16,8-31,6) \\
(3,5-15,3)\end{array}$} \\
\hline Menos de um & 44 & 69,1 & \\
\hline De um a 1,9 & 14 & 23,4 & \\
\hline Dois ou mais & 3 & 7,5 & \\
\hline Percepção de saúde & & & \multirow{4}{*}{$\begin{array}{r}(9,0-73,0) \\
(20,9-77,7) \\
(3,2-55,3)\end{array}$} \\
\hline Muito boa ou boa & 21 & 34,1 & \\
\hline Regular & 32 & 49,0 & \\
\hline Ruim ou muito ruim & 10 & 16,8 & \\
\hline Suspeita de déficit cognitivo & & & \multirow{3}{*}{$\begin{array}{r}(44,2-93,5) \\
(6,5-55,8)\end{array}$} \\
\hline Não & 51 & 77,2 & \\
\hline $\operatorname{Sim}$ & 17 & 22,8 & \\
\hline História de queda alguma vez na vida & & & \multirow{3}{*}{$\begin{array}{l}(14,3-65,0) \\
(34,9-85,7)\end{array}$} \\
\hline Não & 22 & 35,8 & \\
\hline Sim & 46 & 64,2 & \\
\hline Suspeita de depressão & & & \multirow{3}{*}{$\begin{array}{l}(22,3-62,4) \\
(37,6-77,7)\end{array}$} \\
\hline Não & 28 & 40,8 & \\
\hline Sim & 20 & 59,2 & \\
\hline Motivo da restrição à residência e/ou leito & & & \multirow{4}{*}{$\begin{array}{r}(55,4-75,8) \\
(9,6-39,3) \\
(5,9-25,8)\end{array}$} \\
\hline De natureza física & 42 & 66,4 & \\
\hline De natureza cognitivo & 13 & 20,7 & \\
\hline De natureza comportamental & 9 & 12,9 & \\
\hline
\end{tabular}

"Número de idosos da amostra; ** estimado após expansão da amostra; ${ }^{* * *}$ Intervalo de confiança a 95\%, considerando-se a estratificação de acordo com o IVS, as MA como Unidades Primárias de Amostragem e o Fator de Expansão do indivíduo. 
A Tabela 3 apresenta a capacidade de desempenho nas atividades básicas de vida diária dos idosos restritos ao domicílio. Segundo o relato dos entrevistados, a principal atividade básica de vida diária que necessitava de ajuda de outros para sua execução foi a "locomoção". Em relação às AIVDs, como esperado, todos os idosos referiram não serem capazes de ir a lugares distantes sem auxílio. Grande parte deles também referiu necessidade de ajuda para a realização das demais AIVDs, sendo que "fazer compras", "limpar a casa”, "preparar refeição", "ir a lugares distantes” e "lidar com as finanças" se destacam como as que mais necessitam de uma ajuda completa em sua realização. Em relação à classificação da capacidade funcional entre os idosos restritos ao domicílio, a ocorrência de dependência grave (dificuldade em realizar mais de 7 tarefas) foi de 55,1\%, dependência moderada (dificuldade em realizar mais de 4-6 tarefas) de 42\% e a dependência leve (dificuldades em realizar 1-3 tarefas) apenas 2,9\%.

A Tabela 4 indica a prevalência de restrição de domicílio de acordo com características sociodemográficas e de saúde. Como pode ser observado, a prevalência da condição foi maior entre as mulheres, entre os indivíduos com 80 anos ou mais e entre aqueles com suspeita de déficit cognitivo. Ao se calcular a razão de prevalência entre os subgrupos, nota-se que a prevalência de restrição foi 1,54 vez maior entre as mulheres quan- do comparadas aos homens; 2,63 vezes maior entre os com suspeita de déficit cognitivo quando comparados aos negativos; e cerca de 4 vezes maior entre os maiores de 80 anos, quando comparados aos com idade entre 60 e 69. Nota-se também que esta foi superior entre os com menor escolaridade e com maior risco social, porém essas diferenças não se mostraram estatisticamente significativas.

\section{Discussão}

Como descrito na seção anterior, cerca de $1 / 5$ dos idosos entrevistados se referiram ser restritos ao domicílio. Tal prevalência é superior à observada em estudos populacionais realizados no Japão, que indicam uma variabilidade entre $3,3 \%$ e $7,7 \% 0^{3,6,7}$. As diferenças entre a prevalência estimada neste estudo e as provenientes dos inquéritos internacionais poderiam ser justificadas por vários fatores, dentre os quais se destacam aqueles relativos às diferenças conceituais da condição estudada nas diferentes pesquisas e os relativos ao perfil heterogêneo das amostras.

Em relação ao conceito atribuído à restrição ao domicílio, dada a escassez de estudos nacionais sobre o tema, foi realizada uma comparação com estudos japoneses, ressaltando-se as diferenças de definições entre as amostras. Shinkai

Tabela 3. Distribuição dos idosos restritos ao domicílio e/ou ao leito segundo o desempenho nas ABVD e AIVD - Belo Horizonte (MG, Brasil), 2006.

\begin{tabular}{|c|c|c|c|c|c|c|}
\hline \multirow[b]{2}{*}{ Atividades } & \multicolumn{2}{|r|}{ Sem ajuda } & \multicolumn{2}{|c|}{ Ajuda parcial } & \multicolumn{2}{|r|}{ Ajuda total } \\
\hline & $\mathbf{n}$ & $\%^{*}$ IC $(95 \%)^{* *}$ & $\mathbf{n}$ & $\%^{*}(\text { IC } 95 \%)^{* *}$ & $\mathbf{n}$ & $\%^{*}(\text { IC } 95 \%)^{* *}$ \\
\hline \multicolumn{7}{|l|}{ ABVD } \\
\hline Comer & 54 & $78,9(54,8-92,0)$ & 10 & $12,9(2,2-49,0)$ & 4 & $8,2(1,4-35,4)$ \\
\hline Vestir-se & 48 & $64,5(37,7-84,7)$ & 13 & $23,3(7,9-51,7)$ & 7 & $12,1(3,8-32,5)$ \\
\hline Pentear cabelo & 52 & $72,8(58,7-83,5)$ & 4 & $8,6(3,1-21,9)$ & 12 & $18,5(11,0-29,5)$ \\
\hline Locomover-se & 39 & $13,8(4,1-37,2)$ & 21 & $31,5(12,1-60,4)$ & 8 & $13,8(4,1-37,3)$ \\
\hline Deitar/levantar da cama & 55 & $78,9(68,9 \quad 86,3)$ & 6 & $9,0(1,6-37,9)$ & 7 & $12,1(3,8-32,5)$ \\
\hline Tomar banho & 43 & $55,2(30,0 \quad 78,0)$ & 17 & $31,0(10,6-63,0)$ & 8 & $13,8(4,1-37,3)$ \\
\hline Ir ao banheiro em tempo & 37 & $56,9(44,8-68,2)$ & 28 & $38,4(23,9-55,1)$ & 3 & $4,7(0,8-22,9)$ \\
\hline \multicolumn{7}{|l|}{ AIVD } \\
\hline Uso do telefone & 11 & $19,0(8,6-36,8)$ & 36 & $47,8(36,8-59,1)$ & 21 & $33,2(22,7-45,7)$ \\
\hline Ir a lugares distantes & 0 & - & 43 & $54,7(22,9-83,1)$ & 25 & $45,3(16,9-77,1)$ \\
\hline Fazer compras & 1 & $2,5(0,1-28,6)$ & 21 & $26,7(11,9-49,5)$ & 46 & $71,1(51,9-84,9)$ \\
\hline Preparar refeição & 22 & $30,2(18,7 \quad 44,9)$ & 12 & $16,8(6,3-38,0)$ & 34 & $53,0(32,1-72,9)$ \\
\hline Limpar casa & 14 & $19,8(5,0-53,6)$ & 14 & $18,5(4,4-52,8)$ & 40 & $61,6(43,6-76,9)$ \\
\hline Tomar medicamento & 29 & $44,8(29,9-60,8)$ & 24 & $31,5(14,3-55,8)$ & 15 & $23,7(11,1-43,8)$ \\
\hline Lidar com finanças & 2 & $4,3(0,2-46,7)$ & 40 & $54,7(43,0-66,0)$ & 26 & $40,9(24,1-60,2)$ \\
\hline
\end{tabular}

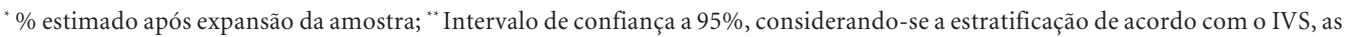
microáreas como Unidades Primárias de Amostragem (UPA) e o fator de expansão de cada indivíduo. 
Tabela 4. Prevalência de idosos restritos ao domicílio e/ou leito em subgrupos populacionais de acordo com características sociodemográficas e de saúde - Belo Horizonte (MG, Brasil), 2006.

\begin{tabular}{llc}
\hline \multirow{2}{*}{$\begin{array}{c}\text { Características } \\
\text { sociodemográficas e de saúde }\end{array}$} & \multicolumn{2}{c}{$\begin{array}{c}\text { Restritos ao domicílio } \\
\text { e/ou ao leito }\end{array}$} \\
\cline { 2 - 2 } & $\mathbf{n} \%^{*}$ & $(\mathbf{I C 9 5} \%)^{* *}$ \\
\hline Sexo & & \\
$\quad$ Feminino & 25,6 & $(16,9-36,9)$ \\
Masculino & 16,7 & $(10,3-25,9)$ \\
p-valor & $\mathbf{0 , 0 1 5}$ & \\
Idade & & \\
De 60 a 69 anos & 14,4 & $(7,6-25,6)$ \\
De 70 a 79 anos & 16,0 & $(7,9-29,7)$ \\
80 anos ou mais & 57,0 & $(25,0-84,0)$ \\
p-valor & $\mathbf{0 , 0 0 8}$ & \\
Anos completos de escolaridade & & \\
Até sete anos de estudo & 24,5 & $(13,6-39,9)$ \\
Oito anos ou mais & 13,3 & $(8,1-21,2)$ \\
p-valor & $\mathbf{0 , 1 1 9}$ & \\
Suspeita de déficit cognitivo & & \\
Não & 19,2 & $(11,5-30,3)$ \\
Sim & 50,5 & $(24,5-76,2)$ \\
p-valor & $\mathbf{0 , 0 0 5}$ & \\
Indice de Vulnerabilidade & & \\
Baixo/Médio/Elevado risco & 30,5 & $(19,6-44,1)$ \\
Muito elevado risco & 53,7 & $(19,3-84,9)$ \\
p-valor & $\mathbf{0 , 1 7 3}$ & \\
\hline
\end{tabular}

*\% estimado após expansão da amostra; ${ }^{* *}$ Intervalo de confiança a $95 \%$, considerando-se a estratificação de acordo com o IVS, as microáreas como Unidades Primárias de Amostragem (UPA) e o fator de expansão de cada indivíduo.

et al. ${ }^{7}$ consideram idosos restritos ao domicílio aqueles que saem de casa apenas uma vez por semana ou menos que isso, classificando-os em tipo 1 (idosos que não são capazes de sair de casa sem auxilio) e tipo 2 (idosos que saem apenas na vizinhança sem auxílio). Neste estudo, foi encontrada uma prevalência de idosos restritos ao domicílio tipo 1 de 4,1\% na cidade de Yoita e 3,3\% em Hatoyama. No estudo de Haraguchi et al. ${ }^{6}$, o conceito de idoso restrito ao domicílio está associado à incapacidade de sair de casa independentemente, o que se assemelha ao conceito utilizado no presente estudo. Já para Imuta et al. ${ }^{3}$, idosos restritos ao domicílio são pessoas cuja realização das atividades de vida diária está limitada ao círculo doméstico, o que não significa incapacidade de realizá-las em ambiente comunitário. Verifica-se um conceito mais abrangente de restrição ao domicílio utilizado pela literatura internacional, o que nos levaria a esperar uma preva- lência mais alta nessa população do que a observada na área de abrangência do CSV, o que, de fato, não aconteceu.

Outra questão dessa natureza a ser considerada diz respeito à definição operacional do indivíduo idoso. Observa-se que há uma tendência à classificação do idoso como sendo os indivíduos com idade superior a 65 anos na literatura internacional ${ }^{19}$, contrastando-se ao ponto de corte utilizado neste estudo (60 anos). Entretanto, estas diferenças também nos fariam crer que a prevalência de restrição ao domicílio no presente estudo seria inferior à encontrada em pesquisas que incluem apenas os indivíduos mais velhos, já que estes, em razão do acúmulo de patologias e de incapacidades próprias do envelhecimento, potencialmente teriam menor mobilidade.

Diante do exposto, a maior prevalência de restrição ao domicílio no presente estudo não parece ser proveniente de diferenças de cunho metodológico, mas sim ser justificada pelas diferenças no perfil de saúde, socioeconômico e cultural entre os idosos residentes na área de abrangência do Centro de Saúde Ventosa e os residentes nos países mais desenvolvidos, como o Japão. Boa parte dos idosos brasileiros apresenta precárias condições de vida, em que escassez de recursos financeiros é somada à superposição de patologias e dificuldades de acesso aos serviços de saúde de maior complexidade. Somam-se a isto as dificuldades nos serviços de infraestrutura urbanos, dificultando ainda mais a autonomia do idoso.

Como descrito na seção Resultados, a análise dos motivos alegados para a restrição ao domicílio indica que o principal fator de limitação parece ter sido a dependência física, resultante em sua maioria de um AVE anterior ou de alterações na acuidade visual. Essa evidência não é exclusividade da população estudada. Tanto a literatura internacional como a nacional apontam que esses problemas são considerados significativos fatores de risco para a incapacidade funcional. Em estudo nacional realizado no estado de São Paulo, Rosa et al..$^{5}$ verificaram um risco 7,8 vezes maior de desenvolver dependência moderada/ grave entre os idosos com história de AVE. Já em estudo realizado com idosos no estado do Paraná, os problemas relativos à acuidade visual foram citados como os que mais impossibilitavam $\mathrm{o}$ idoso de se locomover para fora de casa ${ }^{20}$.

O predomínio de idosas entre os indivíduos restritos ao domicílio também não deve ser visto como uma característica exclusiva dessa população. Outros estudos com idosos brasileiros já 
apontaram a distribuição desigual entre os gêneros a partir de uma certa faixa etária ${ }^{16,21}$. As mulheres, por serem mais longevas que os homens, tendem a apresentar mais incapacidades do que eles, que acabam por restringir sua circulação ao domicílio.

Também merece debate o perfil dos idosos restritos ao domicílio com relação ao seu arranjo familiar. Assim como em estudos nacionais anteriores, a proporção de idosos restritos que residem com duas ou mais gerações foi significativamente maior do que os que convivem apenas com indivíduos de uma única geração ${ }^{16,21}$. Duarte $e t$ al. ${ }^{22}$ destacam a presença dos filhos nos arranjos domiciliares, pois representam o maior potencial de auxílio aos idosos, agindo, dessa forma, como fator de proteção. Esses indivíduos mais jovens compõem uma rede social fundamental para o idoso, podendo se constituir em importantes aliados das equipes de saúde da família no planejamento das ações.

Grande parte da população restrita ao domicílio apresentou baixo nível de escolaridade e de renda. Esses achados também concordam com os apresentados por Rosa et al. ${ }^{5}$. O reconhecimento desse perfil socioeconômico dos idosos restritos também é fundamental para a elaboração de propostas efetivas de promoção de saúde, já que, dentre outros aspectos, essa condição social dificulta a conscientização das pessoas para a necessidade de cuidado com a saúde, a adesão ao tratamento e a manutenção do estilo de vida saudável, limitando as ações dos profissionais de saúde.

Outro aspecto que merece discussão diz respeito à autoavaliação da condição de saúde. Dentre os entrevistados, a maioria percebe sua saúde como "regular". Tal proporção é maior do que a encontrada por Lima-Costa et al. ${ }^{21} \mathrm{em}$ uma amostra representativa da população brasileira de idosos. A diferença pode ser explicada pelo alto grau de dependência nas AVDs dos idosos restritos ao domicílio, fator fortemente relacionado à autopercepção de saúde ${ }^{14}$. Vale ressaltar que, por sua facilidade de investigação, alta correlação com o real estado de saúde e por ser um forte preditor de mortalidade e declínio funcional, parece bastante oportuno que essa avaliação seja incorporada à rotina dos profissionais da atenção básica.

Dentre as patologias habitualmente presentes em indivíduos idosos, a hipertensão arterial sistêmica (HAS) foi o agravo mais frequentemente relatado pelos restritos ao domicílio. No entanto, como apontado anteriormente, o AVE, os problemas de coluna, de memória e a incontinência urinária se constituem as doenças que mais interferem em suas atividades diárias. Tendo em vista a possibilidade de postergar a redução da independência do idoso, os achados sugerem fortemente que estratégias de promoção de saúde que previnam o aparecimento dessas condições, bem como as que visem à detecção precoce delas e seu tratamento, também devem ser priorizadas pelas equipes de saúde da família.

A alta prevalência de quedas entre os indivíduos restritos ao domicílio também chama a atenção. Como era de se esperar, as prevalências descritas no presente estudo são bem superiores às relatadas em estudos realizados com idosos da comunidade ${ }^{20}$. É possível que essa maior suscetibilidade às quedas e fraturas possa ser explicada pela maior limitação físico-funcional em relação aos idosos da comunidade em geral. A alta prevalência de idosos com suspeita de depressão também deve ser comentada. Taxas semelhantes são descritas em estudos com idosos institucionalizados no Brasil ${ }^{23}$. Essa alta prevalência de depressão encontrada nos idosos restritos revela a sua importância para a saúde pública, tanto no sentido de estimular os profissionais na identificação de casos como no de garantir o tratamento adequado a essa população.

Quanto às atividades básicas de vida diária, de modo geral, os resultados da pesquisa são semelhantes aos reportados em estudos anteriores. Trelha et al. ${ }^{24}$, ao avaliarem a capacidade funcional de idosos restritos ao domicílio de uma unidade básica de saúde em Londrina (Paraná), também identificaram uma alta frequência de idosos que referiam grande dificuldade em ir ao banheiro em tempo, tomar banho e se locomover sem o auxílio de outros. Tal evidência também foi descrita por Imuta et al. ${ }^{3}$, que identificaram as dificuldades na locomoção e na higienização como importantes fatores de risco para a restrição ao domicílio.

Chama a atenção a grande proporção de idosos restritos que apresenta dependência grave com dificuldade na realização de sete ou mais AVDs, uma vez que as estimativas são superiores às apresentadas em estudos anteriores com idosos da comunidade ${ }^{5,16}$. Essa maior proporção de dependência funcional grave entre os idosos deste estudo não é nenhuma surpresa, já que se trata de uma amostra de indivíduos restritos ao domicílio de faixa etária elevada, com diversas patologias associadas e com baixo nível de escolaridade e renda, reconhecidos fatores de risco para dependência funcional ${ }^{4,5}$.

A prevalência da condição de restrição foi significativamente maior entre as mulheres, indiví- 
duos com 80 anos ou mais e entre aqueles com suspeita de déficit cognitivo, corroborando os achados de estudos realizados com idosos restritos ao domicílio ${ }^{6,7,19}$. Shinkai et al. ${ }^{7}$ verificaram um percentual maior de idosos restritos ao domicilio com o avançar da idade e uma menor prevalência entre aqueles com atividade intelectual alta. Para Ganguli et al. ${ }^{19}$ e Haraguchi et al. ${ }^{6}$, a idade e o gênero feminino são fatores significativamente associados a essa condição.

Observaram-se também diferenças importantes entre as prevalências de restrição ao domicílio nas categorias escolaridade e IVS, porém estas não foram significantes. É possível que isso tenha ocorrido em razão do pequeno tamanho amostral. Fazem-se necessários, portanto, estudos futuros com maior tamanho amostral que abordem o tema de restrição ao domicílio entre os idosos. Ao se discutir a distribuição das ocorrências de restrição nos diferentes subgrupos populacionais, vale ressaltar que, dado o seu delineamento transversal, este estudo não teve como objetivo definir o caráter etiológico da restrição ao domicílio, e sim prover um conhecimento inédito em relação à prevalência e ao perfil de idosos restritos ao domicílio no contexto da realidade brasileira.

\section{Conclusão}

Apesar da ausência de estudos que permitam comparações entre diferentes regiões brasileiras e da impossibilidade de generalização dos resultados, acredita-se que a alta prevalência de idosos restritos ao domicílio dentre os idosos adstritos ao PSF/Ventosa de Belo Horizonte não seja um fato isolado e que esse cenário possa ser encontrado em outras comunidades com características demográficas, culturais e socioeconômicas semelhantes.

Considerando a cobertura cada vez maior do Programa Saúde da Família em todo o Brasil, este parece ser uma ferramenta estratégica para o enfrentamento do problema, possibilitando uma adequada atenção a esse grupo de idosos. Uma investigação permanente da presença de idosos que não frequentem a unidade de saúde, seja através das visitas domiciliares ou de outras ações que visem à identificação deles, poderia se constituir na única maneira de dar visibilidade a esse idoso que, por vezes, permanece em seu lar, invisível ao sistema de saúde.

\section{Colaboradores}

PGS Ursine participou do delineamento do estudo, análise dos dados, discussão dos resultados e foi a redatora principal do artigo; HA Cordeiro orientou o delineamento do estudo e realizou a revisão da redação; CL Moraes colaborou no delineamento do estudo, análise dos dados e revisão crítica final do artigo. 


\section{Referências}

1. Ramos LR. Growing old in São Paulo, Brazil: assessment of health status and family suppor of elderly of different socio-economic strats living in the community [tese]. Londres: University of London; 1987.

2. Fujita K, Fujiwara Y, Chaves PHM, Motohashi Y, Shinkai S. Frequence of going outdoors as a good predictors for incidence disability of physical function as well as disability recovery in communitydwelling older adults in rural Japan. J Epidemiology 2006; 16(6):261-270.

3. Imuta $H$, Yasumura S, Fujita $M$, Arai H, Fukao A. Homebound elderly in a Japanese community: related factors and change of mobility. Nippon Koshu Eisei Zasshi 1998; 45(9):883-892.

4. Giacomin KC, Uchoa E, Firmo JOA, Lima-Costa MF. Projeto Bambuí: um estudo de base populacional da prevalência e dos fatores associados à necessidade de cuidador entre idosos. Cad Saude Publica 2005; 21(1):80-91.

5. Rosa TEC, Benício HA, Latorre MRD, Ramos LR. Fatores determinantes da capacidade funcional entre idosos. Rev Saude Publica 2003; 37(1):40-48.

6. Haraguchi Y, Osaki Y, Kishimoto T, Yakura N. Prevalence of the homebound in the elderly population within a community determined by using three measurement scales. Nippon Eiseigaku Zasshi 2006; 61(1):44-52.

7. Shinkai S, Fujita K, Fujiwara Y. Prevalence and characteristics of different types of homeboudness among community-living older adults. Nippon Koshu Eisei Zasshi 2005; 52(7):443-455.

8. Brasil. Ministério da Saúde. Secretaria de Assistência à Saúde. Saúde da Família: uma estratégia para a reorientação do modelo assistencial. Brasília. Ministério da Saúde; 1998.

9. Ramos LR, Veras RP, Kalache A. Envelhecimento populacional: uma realidade brasileira. Rev Saude Public 1987; 21(3):211-224.

10. Belo Horizonte. Secretaria Municipal de Saúde. Plano Municipal de Saúde de Belo Horizonte 20052008. Belo Horizonte: Secretaria Municipal de Saúde; 2005.

11. Veras RP, Silva SD, Souza CAM, Milioli R, Ventura F. Proposta metodológica para inquérito domiciliar com populações idosas em um centro urbano do Estado do Rio de Janeiro (Brasil). Rev Saude Publica1989; 23(5):429-438.

12. Folstein MF, Folstein SE, Mchugh, PR. Mini-mental state: a practical method for grading the cognitive state of patients for the clinician. J Psychiatric Research 1975; 12(3):189-198.

13. Baltes MM. The many faces of dependency in old age. Cambrige: Cambrige University Press; 1996.

14. Alves LC, Rodrigues, RN. Determinantes da autopercepção de saúde entre idosos do município de São Paulo, Brasil. Rev Panam Salud Publica 2005; 17(5/6):333-341.
15. Duke University Center for the Study of Aging and Human Development. Multidimensional functional assessment: the Oars methodology. Durhan: Duke University Center for the Study of Aging and Human Development; 1978.

16. Ramos LR, Rosa TE, Oliveira ZM, Medina MCG, Santos FRG. Perfil do idoso em área metropolitana na Região Sudeste do Brasil: resultados de inquérito domiciliar. Rev Saude Publica 1993; 27(2):87-94.

17. Sheikh JI, Yesavage JA. Geriatric Depression Scale (GDS): recent evidence and development of a short version. Clin Gerontol 1986; 5:165-173.

18. StataCorp. Stata Statistical Software: release 9. College Station (TX) [programa de computador]. Texas: Stata Corporation; 2005.

19. Ganguli M, Fox A, Gilby J, Belle S. Characteristics of rural homebound older adults: a community based study. J Am Geriatric Society 1996; 44(4):363-370.

20. Farinasso ALC. Perfil dos idosos em uma área de abrangência da Estratégia Saúde da Família [dissertação]. Ribeirão Preto: Escola de Enfermagem de Ribeirão Preto, Universidade de São Paulo; 2004.

21. Lima-Costa MF, Barreto SM, Giatti L. Condições de saúde, capacidade funcional, uso de serviços de saúde e gastos com medicamento da população brasileira: um estudo descritivo baseado na Pesquisa Nacional por Amostra de Domicílios.Cad Saude Publica 2003; 19(3):735-743.

22. Duarte YAO, Lebrão ML, Lima FD. Contribuição dos arranjos domiciliares para o suprimento de demandas assistenciais dos idosos com comprometimento funcional em São Paulo, Brasil. Rev Panam Salud Publica 2005; 17(5/6):370-378.

23. Cheloni CFP, Pinheiro FLS, Filho MC, Medeiros AL. Prevalência de depressão em idosos institucionalizados no município de Mossoró/RN segundo escala de depressão geriátrica. Expressão, Mossoró 2003; 34(1/2):61-73.

24. Trelha CS, Nakaoski T, Franco SS, Dellarosa MSG, Yamada KN, Cabrera M, Mesas AE, Gaetan CCB. Capacidade funcional de idosos restritos ao domicílio do Conjunto Ruy Virmond Carnascialli, Londrina/PR. Semina: Ciências Biológicas e da Saúde 2005; 26(1):37-46.

Artigo apresentado em 04/11/2008

Aprovado em 24/04/2009

Versão final apresentada em 30/05/2009 